\title{
Strong Coupled Agglomeration Multigrid for Navier-Stokes Equations on Unstructured Grids
}

\author{
Zongzhe Li, Zhenghua Wang, Wei Cao, Lu Yao \\ School of Computer Science \\ National University of Defence Technology \\ Changsha, Hunan 410073, P.R.China
}

\begin{abstract}
This paper focused on a series of agglomeration multigrid techniques for the numerical solution of the 2D/3D Reynolds Averaged Navier-Stokes equations on cell-centered unstructured grids, which conform with FVM discretization scheme. We explore a new agglomeration strategy to automated generate coarse grids for multigrid methods, which can conduct to 2D/3D unstructured/hybrid grids. The algorithm based on face weighting, fuse the strongest coupled neigh-bor face to form a new cell in coarsen grid once at a time. The numerical results which conduct on NACA0012 airfoil indicate that nearly optimal computational complexity, this method also shows better complexity than the listed method owing to the better quality of the coarse grid.
\end{abstract}

Keywords-Strong coupled agglomeration, Unstructured grids, Cell-centered, Multigrid.

\section{INTRODUCTION}

The development of robust and efficient algorithms for explicit and implicit solution of Reynolds Averaged NavierStokes equations in unstructured grids is an active research topic. Multigrid methods are among the fastest numerical algorithms for the solution of large sparse systems equations. A key issue for using multigrid is the generation of the coarse grid levels.

Over the last few years, three different approaches can be adopted for generate unstructured multilevel girds process. The first one begins with a coarse mesh to generate finer grid by refinement [1]; the second approach uses nonnested unstructured grids as coarse grids $[2,3]$, which are generated independently using any given gird generation strategy; For complex geometries, especially in $3 \mathrm{D}$, the above two methods construct coarse grids that faithfully represent the complex geometries can become a difficult proposition. The third to generate coarse grids through agglomeration, which is first introduced by Lallemand [4] and Smith [5], circumvents this problem by using heuristics to fuse the control volumes for the cell or vertex. Several improvements [6-9] have been made to agglomeration grids in order to optimize the fused cells according to a surface to volume ratio and coarsening ratio between different grid levels. Other improvements [10,11] need additional knowledge about the cell types of the primary grid for the agglomerated volumes. For inviscid transonic flow, the theoretically convergence rate for isotropic agglomeration multigrid can be achieved. However. for high Reynolds number Navier-Stokes problems with highly stretched grids, the efficiency of multigrid will broken down. Mavriplis [12] has demonstrate an algorithm coupling with directional- coarsening and line-implicit smoothing to improve viscous conver-gence rate. Directional agglomeration restrict merging directions with edge coefficients on highly stretched grids. Many reference [12-15] demonstrate the best features for directional-coarsening grid generator and the method in present work is also inspired by this techniques.

In this manuscript, we develop a heuristic strong coupled agglomeration technique, designed for cell-centered control volumes and finite volume scheme multigrid method, which can produce better quality in coarse grid shape for 2D and can be easily extended to 3D. Finally, the approach is compared with the isotropic [4], surface to volume ratio [6] based improvement and directional coarsened [12] multigrid algorithm for solving Navier-Stokes equations, which provide better conver-gence acceleration.

\section{GOVERNING EQUATIONS AND DISCRETIZATION}

The Reynolds Averaged Navier-Stokes equations in integral form for 3D compressible steady flows can be expressed as

$$
\frac{\partial}{\partial t} \iiint \mathbf{Q} \cdot d \mathbf{V}+\oiint\left(\mathbf{F}_{i}-\mathbf{G}_{i}\right) \cdot n_{i} d \mathbf{S}=0
$$

Where $\mathbf{V}$ is the control volume, $\mathbf{S}$ is cell's surface area and $\mathbf{n}$ is the unit outward vector normal to the surface area. $\mathbf{Q}$ is the vector of the conservation, $\mathbf{F}$ is inviscid flux vector, $\mathbf{G}$ is viscous flux vector, are defined by

$$
\mathbf{Q}=\left[\begin{array}{l}
\rho \\
\rho u_{1} \\
\rho u_{2} \\
\rho u_{3} \\
e
\end{array}\right], \mathbf{F}_{i}=\left[\begin{array}{c}
\rho u_{i} \\
\rho u_{1} u_{i}+p \delta_{1 i} \\
\rho u_{2} u_{i}+p \delta_{2 i} \\
\rho u_{3} u_{i}+p \delta_{3 i} \\
(e+p) u_{i}
\end{array}\right], \mathbf{G}_{i}=\left[\begin{array}{c}
\rho u_{i} \\
\tau_{1 i} \\
\tau_{2 i} \\
\tau_{3 i} \\
u_{k} \tau_{k i}-q_{i}
\end{array}\right]
$$

Here $\rho, p, e$ denote the density, pressure, total energy, respectively, $\delta_{i j}$ is the Kronecker delta and $u_{i}$ is the velocity of the flow in the coordinate direction $x_{i}$. the corresponding viscous stress tensor and the averaged heat flux is defined by

$$
\tau_{i j}=-\frac{2}{3} \mu \frac{\partial u_{k}}{\partial x_{k}} \delta_{i j}+\mu\left(\frac{\partial u_{i}}{\partial x_{j}}+\frac{\partial u_{j}}{\partial x_{i}}\right), q_{i}=-k \frac{\partial T}{\partial x_{i}}
$$

Here $T$ is the temperature of the fluid, $k$ is coefficient of heat exchange, $\mu$ is the viscosity coefficient. Using CellCentered type system, equation (1) can be re-written in semi-discrete form as.

$$
\frac{\Delta \mathbf{Q}_{i}{ }^{n}}{\Delta t} V_{i}=-\sum_{j=1}^{n f}[(\overline{\mathbf{F}}-\overline{\mathbf{G}}) \Delta \bar{s}]_{j}^{n+1}=-\mathbf{R}^{n+1}
$$


Where $n f$ is total faces number of the cell $i, \overline{\mathbf{F}}$ and $\overline{\mathbf{G}}$ are spatially discretized inviscid flux vector and viscous flux vector, $\bar{s}$ is normal area of the cell face. Equation (4) can be linearized in time as

$$
\begin{aligned}
& \left(\frac{V_{i}}{\Delta t} \mathbf{I}+\sum_{j=1}^{n f}\left(\frac{\partial \mathbf{R}^{n}}{\partial \mathbf{Q}} \cdot \bar{s}\right)_{j}\right) \Delta \mathbf{Q}_{i}^{n} \\
& +\sum_{j=1}^{n f}\left(\frac{\partial \mathbf{R}^{n}}{\partial \mathbf{Q}} \cdot \bar{s}_{i j} \cdot \Delta \mathbf{Q}_{j}^{n}\right)=-\mathbf{R}^{n}
\end{aligned}
$$

\section{Strong Coupled Agglomeration Method}

For equation (5), high Reynolds number Navier-Stokes problems with high stretched grids, which conducts huge difference in the coefficient of $\Delta \mathbf{Q}_{j}^{n}$. In the algebraic multigrid sense, which conduct anisotropic problems, will reduce the efficiency of isotropic agglomeration. Directional coarsening techniques can be considered for anisotropic problem involving highly stretched meshes, However, the memory usage of a directional coarsening combine with fully implicit solver grows rapidly with an increasing number of nodes, particularly in 3D. Except this, there will be more than one direction with the same weight, the user may be required to determine the merge direction.

The present agglomeration algorithm uses a face weight take absolute value of face normal area $\overline{s_{i j}}$ between cell $i$ and cell $j$, which represents the degree of coupling in the discretization. For cell-centered finite volume scheme, this coarsening algorithm can be given as follows.

Algorithm 1 (Strong Coupled Agglomeration)

Input: Primary grid in cell set $\mathrm{C}$

Strong/weak coupled threshold $\Gamma$

Output: Coarsen grid in cell set $\mathrm{G}$

Initialization: For all $i$ computing each face weight $w_{i j}$

For all $i, S_{i}=\left\{j \in C \backslash i \mid w_{i j}>\Gamma \max w_{i k}\right\}$

Sort set $\mathrm{C}$ by wall, far field, inner cell.

Algorithm: While $C \neq \varnothing$ do

$$
\begin{aligned}
& \text { Select } i \in C \text {, set } N_{i}=\left\{j \in C \backslash i \mid \exists w_{i j}\right\} \\
& \text { Select } j \in N_{i} \text { and } w_{i j}=\max _{k \in N_{i}} w_{i k} \\
& \text { If } j \in S_{i}, G=G \bigcup\{(i, j)\} \\
& \text { Otherwise, } G=G \bigcup\{(i)\} \\
& \text { Update, } C=C \backslash G
\end{aligned}
$$

In 2D ideally each coarse grid cell will be made up of exactly four cells, this approach will repeat in $2 \mathrm{D}$ to achieve favorite optimal performance of multigrid methods. The choice of selecting cell $i \in C$ is frontal type, begin with one of solid wall boundary, which we can choose the smallest volume cell, second by far field boundary. For singletons, which will fuse with its coarse neighbors. One efficient strategy for this process is agglomerating with the smallest coarsening ratio neighbor. If more than one candidate remains, the singleton agglomerate to the coarse grid with the smallest volume.

\section{RESUlTS}

We implement the present algorithm and compare with three agglomeration strategies. The notation is:

Strategy I: The original algorithm which developed by Lallemand et al [4].

Strategy II: The geometrical algorithm developed by Venkatakrishnan and Mavriplis [6].

Strategy III: The directional coarsening developed by Mavriplis [12].

Strategy IV: The present new agglomeration algorithm.

We test four algorithms on NACA0012 airfoil hybrid mesh with 18,501 points, 38,342 faces, 19,841 cells in 2D, which is shown in Figure.1.

Four agglomeration approaches can produce four different types coarse levels. Figure.2-5 show three levels coarse grid produced by four algorithms.

Strategy I implies that agglomerated cell is direct neighbours of the seed cell to produce a new coarsen grid cell. Strategy II implies that less stretched out control volumes are generated. Strategy III implies that cell agglomerate with the direct neighbours and anisotropic cell agglomerate with the most coupled cell. Strategy IV implies that the seed cell agglomerate with the most strongest coupled direct neighbour cell, the next seed selected by the frontal of the last choose one.

As we know all, each coarse grid cell will be made up of exactly four fine grid cells in 2D ideally. Figure.6 depict the frequency distribution of the number of coarsen control volumes versus the coarsening ratio for four strategies.

It is clear that Strategy I and Strategy III give poor control of the coarsening ratio, Strategy II and Strategy IV have a similar dispersion of the coarsening ratio, which all the levels of them with almost the same coarsening ratio. For more details about the four Strategies can refer to the Table I, which $\mathrm{N}$ stand for number of the cells at each levels, respectively, CR stand for the coarsening ratio. However, for Strategy II which produce the coarse grids without considering the anisotropic problems conducted by highly stretched grids, at this point Strategy III and Strategy IV will be very suit for high Reynolds number Navier-Stokes and highly stretched grids problems, but Strategy III have the memory usage grows rapidly with an increasing number of cells, particularly, in 3D situation the shape of cells conduct more complicate direction, So, Strategy IV relatively have the optimal computational complexity

\section{ACKNOWLEDGMENT}

This work is support by the National Basic Research Program of China (No. 2009CB723803). The authors would like to appreciate Prof. L. P. Zhang and PhD X. H. Chang in China Aerodynamics Research and Development Center (CARDC) for their support and help to experiment.

\section{REFERENCES}

[1] J. Peraire, et al., "A 3D finite element multigrid solver for the Euler equations," presented at the AIAA, Aerospace Sciences Meeting and Exhibit, 30th,, Reno, NV, 1992 
[2] D. J. Mavriplis, A. Jameson, "Multigrid Solution of the Two Dimensional Euler Equations on Unstructured Triangular Meshes," AIAA Journal, 1988. 26, PP: 824-831.

[3] D. J. Mavriplis, "Three Dimensional Multigrid for the Euler equations," presented at the Proc AIAA 10th Comp. Fluid Dynamics Conf., Honolulu, Hawaii, 1991.

[4] M. H. Lallemand, et al., "Unstructured multigridding by volume agglomeration: Current status," Computers \& Fluids, 1992. 21, PP: 397-433.

[5] W. A. Smith, "Multigrid Solution of Transonic Flow on Unstructured Grids," presented at the Recent Advances and Applications in Computational Fluid Dynamics, Proceedings of the ASME Winter Annual Meeting, Ed. O. Baysal, 1990.

[6] V. Venkatakrishnan, D. J. Mavriplis, "Agglomeration multigrid for the three-dimensional Euler equations," AIAA Journal, 1995. 33,PP: 633-640.

[7] D. Strauss, J. L. F. Azevedo, "On the Development of an Agglomeration Multigrid Solver for Turbulent Flows," J. of the Braz. Soc. of Mech. Sci. \& Eng, 2003. 35, PP:315-324.

[8] N. Okamoto, K. Nakahashi, "A coarse grid generation algorithm for agglomeration multigrid method on unstructured grids," AIAA Journal, 1998
[9] T. Berglind, "An agglomeration algorithm for Navier-Stokes grids," AIAA Journal, vol. 46, 2000.

[10] M. J. Pandya, N. T. Frink, "Agglomeration Multigrid for an Unstructured-Grid Flow Solver," AIAA Journal, 2004.

[11] V. Hannemann, "Structured multigrid agglomeration on a data structure for unstructured meshes," Int. J. Numer. Meth. Fluids, 2002. 40, PP:361-368.

[12] D. J. Mavriplis, "Directional Agglomeration Multigrid Techniques for High-Reynolds-Number Viscous Flows," AIAA Journal, 1999. 37, PP 1222-1230.

[13] D. J. Mavriplis, "Directional Coarsening and Smoothing for Anisotropic Navier-Stokes Problems " Elect. Trans. Numer. Anal., 1997. 6, PP: 182-197,

[14] J. V. Lassaline, D. W. Zingg, "Development of an Agglomeration Multigrid Algorithm with Directional Coarsening," AIAA Journal, 1999.

[15] E. Morano, D. J. Mavriplis, "Coarsening strategies for unstructured multigrid techniques with application to anisotropic problems," SIAM J. Sci. Comput., 1998. 20, PP: 393-415, .

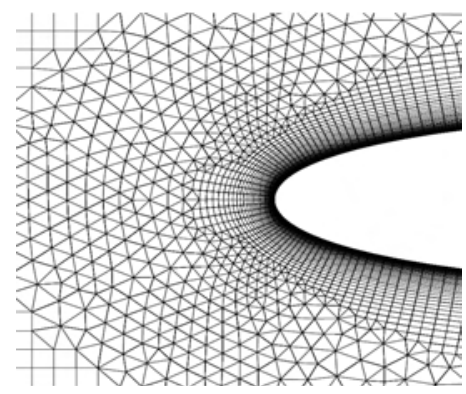

Figure 1. NACA0012 2D airfoil initial hybrid grid

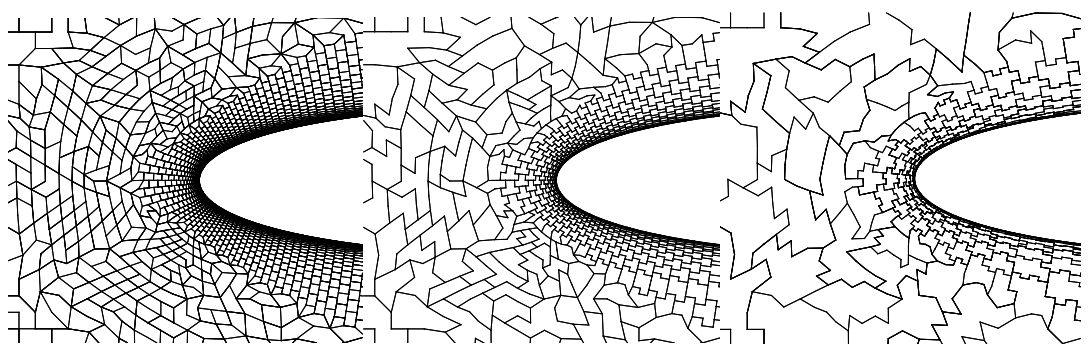

Figure 2. Three levels of coarse grids generated by the agglomeration strategy I

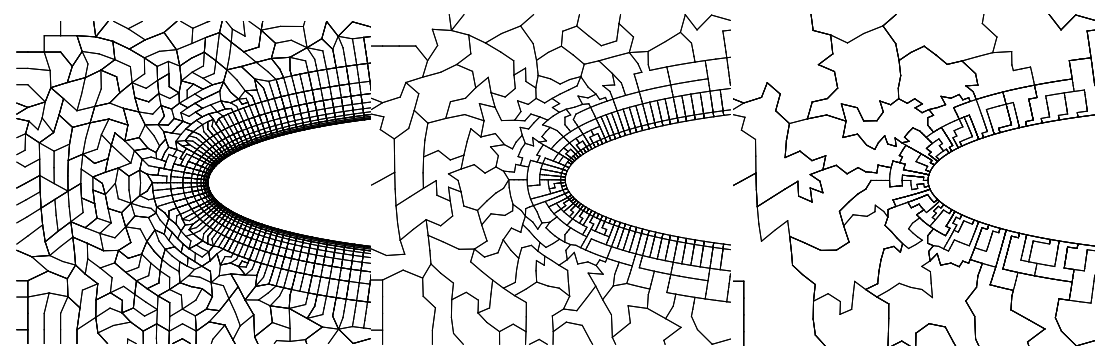

Figure 3. Three levels of coarse grids generated by the agglomeration strategy II 


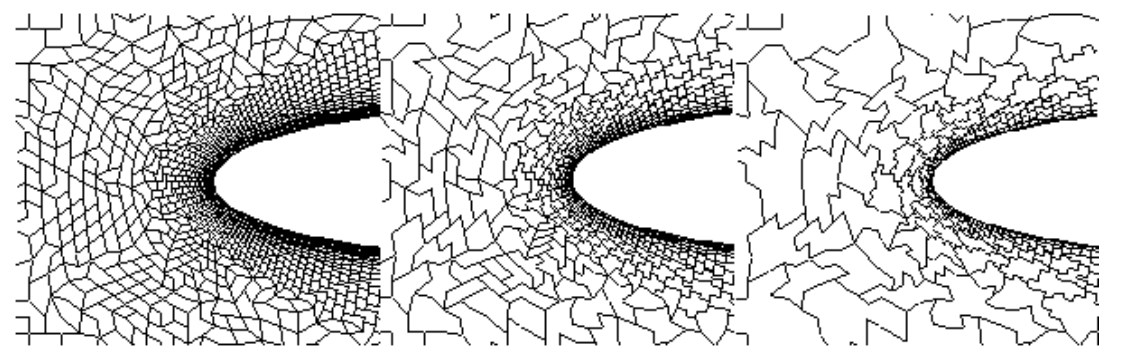

Figure 4. Three levels of coarse grids generated by the agglomeration strategy III

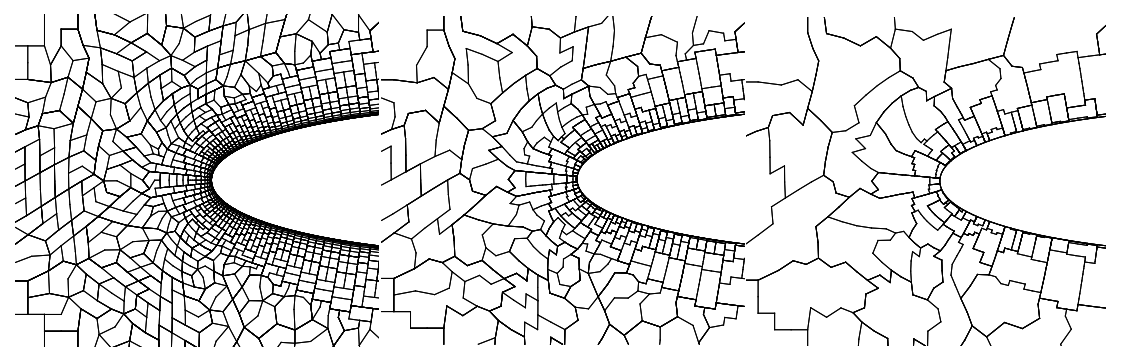

Figure 5 . Three levels of coarse grids generated by the agglomeration strategy IV
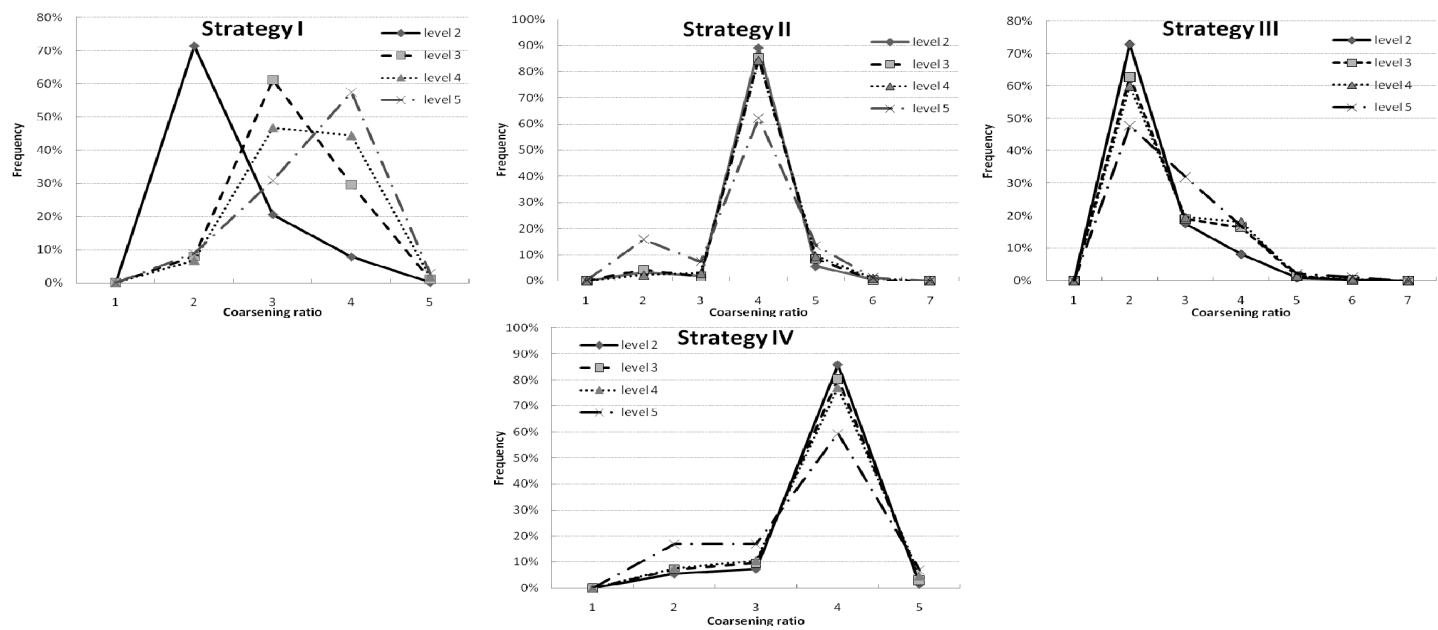

Figure 6. The frequency distribution of the number of coarse control volumes versus the coarsening ratio for four strategies

TABLE I. DETAILS ABOUT FOUR AGGLOMERATION STRATEGIES

\begin{tabular}{ccccccccc}
\hline \multirow{2}{*}{ Levels } & \multicolumn{2}{c}{ Strategy I } & \multicolumn{2}{c}{ Strategy II } & \multicolumn{2}{c}{ Strategy III } & \multicolumn{3}{c}{ Strategy IV } \\
\cline { 2 - 9 } & $\mathrm{N}$ & $\mathrm{CR}$ & $\mathrm{N}$ & $\mathrm{CR}$ & $\mathrm{N}$ & $\mathrm{CR}$ & $\mathrm{N}$ & $\mathrm{CR}$ \\
\hline 2 & 8378 & 2.37 & 4981 & 3.98 & 8331 & 2.38 & 5177 & 3.83 \\
\hline 3 & 2587 & 3.24 & 1248 & 3.99 & 3238 & 2.57 & 1365 & 3.79 \\
\hline 4 & 758 & 3.41 & 309 & 4.04 & 1230 & 2.63 & 360 & 3.79 \\
\hline 5 & 214 & 3.54 & 82 & 3.77 & 444 & 2.77 & 101 & 3.56 \\
\hline
\end{tabular}

